\title{
Políticas de Prevenção e Controle do Câncer Bucal à luz da Teoria da Estruturação de Giddens
}

\author{
Policies for Prevention and Control of Oral Cancer in the light of \\ Giddens' Structuration Theory
}

Fernando Lopes Tavares de Lima (https://orcid.org/0000-0002-8618-7608) ${ }^{1}$

Gisele O'Dwyer (https://orcid.org/0000-0003-0222-1205) ${ }^{2}$

${ }^{1}$ Instituto Nacional de

Câncer José Alencar

Gomes da Silva. Praça Cruz

Vermelha 23, Centro. 20230

130 Rio de Janeiro RJ

Brasil.flima@inca.gov.b

${ }^{2}$ Escola Nacional de Saúde

Pública Sergio Arouca,

Fundação Oswaldo Cruz.

Rio de Janeiro RJ Brasil.

\begin{abstract}
Challenges remain to ensure access to diagnosis and treatment ten years into continuous cancer prevention, control, and oral health policies. This study aims to analyze the oncology and oral health policies in force regarding the process of implanting oral cancer-related care components. Ten policies were analyzed under the lenses of the Structuration Theory, besides data on the supply of services between 2002 and 2017. Low coverage and inadequate regional distribution were highlighted in primary and secondary health care levels, despite increased funding and number of services. Unequal distribution of performed surgeries was identified in tertiary care. The limitation of home care services has hindered users' access to palliative care. A convergence was identified between the analyzed policies and concern with the regulation of authoritative resources and the increase of allocative resources, which stirred the expansion of services. Investments should be made in the expansion, regionalization, and universalization of services. A possible setback in these policies could aggravate the situation and contribute to the increase in health inequalities.
\end{abstract}

Key words Mouth Neoplasms, Health Policy, Oral Health
Resumo Passados mais de dez anos de continuidade das políticas de prevenção e controle do câncer e de saúde bucal, persistem desafios para garantia do acesso ao diagnóstico e tratamento. $O$ objetivo do estudo é analisar as políticas de oncologia e de saúde bucal em vigor, no que se refere ao processo de implantação dos componentes assistenciais relacionados ao câncer bucal. Foram analisadas dez normativas que estruturam essas políticas, sob a ótica da Teoria da Estruturação, além de dados de oferta de serviços entre 2002 e 2017. Nas atenções básica e secundária, destacouse a baixa cobertura assistencial e a distribuição regional inadequada, apesar do aumento do financiamento e do número de serviços. Na atenção terciária foi identificada a distribuição desigual da realização de cirurgias. Por sua vez, a limitação de serviços da atenção domiciliar tem dificultado o acesso dos usuários aos cuidados paliativos. Houve convergência entre as políticas analisadas e uma preocupação com a regulação dos recursos autoritativos e com o aumento de recursos alocativos, o que estimulou a expansão dos serviços. Deve-se investir na ampliação, regionalização e universalização dos serviços. Um possivel retrocesso nessas políticas poderá agravar a situação e contribuir para o aumento das desigualdades em saúde.

Palavras-chave Neoplasias Bucais, Política de Saúde, Saúde Bucal 


\section{Introdução}

O câncer bucal é uma ampla categoria de localizações para neoplasias de diferentes etiologias e perfis histológicos, embora geralmente se refira ao carcinoma epidermóide ${ }^{1}$. Uma revisão de literatura revelou pelo menos 17 termos diferentes que relatam dados sobre câncer bucal, o que tem dificultado a comunicação entre os profissionais². A falta de consenso na definição de câncer bucal é reforçada pela variabilidade na designação anatômica específica da doença em publicações científicas e relatórios ${ }^{2}$. Por não existir uma padronização sobre quais estruturas anatômicas compõem essa classificação, há diferenças entre os sítios incluídos em pesquisas sobre esse assunto, o que dificulta a comparação entre os achados epidemiológicos ${ }^{3}$.

O câncer bucal é a neoplasia maligna mais incidente na região de cabeça e pescoço e tem como principais fatores de risco o uso crônico de tabaco e de álcool, além da exposição ao sol (para câncer de lábio $)^{4,5}$. No ano de 2012 foram estimados cerca de 300 mil novos casos e 145 mil óbitos no mundo ${ }^{6}$. No Brasil a estimativa é de 14.700 casos novos em 2017, sendo 11.200 em homens e 3.500 em mulheres ${ }^{4}$.

Para reduzir a mortalidade e o impacto negativo na qualidade vida é necessário detectar e tratar precocemente essa enfermidade ${ }^{7}$. Porém, embora ocorra em região amplamente acessível ao exame clínico, as lesões ainda são diagnosticadas muito tardiamente ${ }^{8}$. Em estudo nacional, identificou-se que apenas 6,25\% dos tumores são considerados in situ ou estágio I (fase inicial), enquanto os estadiamentos II, III e IV correspondem a $18,19 \%, 34,45 \%$ e $41,12 \%$, respectivamente. Essa situação caracteriza um atraso na linha de cuidado dos usuários com essa neoplasia.

Esse atraso tem sido associado à demora do paciente na busca pelos serviços, com pouca responsabilização do sistema de saúde pelo cuidado integral aos usuários ${ }^{10}$. Estudos que buscaram razões ligadas à organização do sistema de saúde, direcionaram a análise para a programas específi$\cos ^{11}$ ou para a percepção profissional quanto ao problema $^{12,13}$, não relacionando às políticas em vigor e à estrutura disponível.

O enfrentamento do câncer bucal no Brasil tem sido objeto de ações desde os anos de $1930^{5}$. Apesar disso, essas iniciativas eram descontínuas e de pouca abrangência, o que não possibilitou o controle da doença diante dos padrões esperados. Entre os fatores historicamente implicados para esse cenário destacaram-se a subutilização dos serviços pela população, a inadequada formação dos profissionais para diagnóstico do câncer bucal e a valorização dos dentes como únicas estruturas merecedoras de atenção na cavidade bucal ${ }^{14,15}$.

Hoje, as ações públicas de prevenção e o controle do câncer bucal encontram-se na interseção entre a Política Nacional de Prevenção e Controle do Câncer (PNPCC) e a Política Nacional de Saúde Bucal (PNSB). Apesar da ampliação do financiamento, da infraestrutura e dos recursos humanos na área de saúde bucal ${ }^{16}$, ainda existem desafios para o acesso qualificado ao diagnóstico e tratamento pelo Sistema Único de Saúde (SUS). Para superá-los é necessário compreendermos os fatores estruturais relacionados à essas políticas. Assim, o objetivo desse estudo é analisar as políticas de oncologia e de saúde bucal em vigor, no que se refere ao processo de implantação e implementação dos componentes assistenciais relacionados ao câncer bucal.

\section{Metodologia}

Analisou-se as normativas que estruturam a PNPCC e a PNSB identificadas no site da Secretaria de Atenção à Saúde do Ministério da Saúde e localizadas no sistema Saúde Legis. Os dados foram coletados em novembro de 2017. Foram incluídas as que explicitam os componentes assistenciais responsáveis pelo cuidado dos usuários com câncer bucal e excluídas as que não abordam de forma direta a estruturação do cuidado assistencial dessa enfermidade, como por exemplo as políticas de redução do tabagismo, que apesar da importância no controle dessa neoplasia, merecem uma análise específica.

A análise foi complementada com dados sobre o processo de implantação dos componentes assistenciais previstos na PNPCC. Para análise da atenção básica foram apresentadas, por região brasileira, as taxas de variação do número de habitantes, de equipes da Estratégia de Saúde da Família (ESF), de Equipes de Saúde Bucal (ESB), da cobertura populacional dessas equipes e do número de municípios atendidos por essa estratégia. Os dados foram coletados no site da Sala de Apoio à Gestão Estratégica do Ministério da Saúde (SAGE). As taxas de variação foram calculadas pela divisão dos valores encontrados em 2017 pelo de 2002 (maior faixa temporal disponível na SAGE). A mesma lógica de análise foi utilizada para a atenção secundária. As taxas de variação foram calculadas pela divisão dos valores encontrados em 2016 pelo de 2004 (maior faixa temporal disponível na SAGE). 
No âmbito da atenção terciária, foram analisadas, por região brasileira e por tipo de unidade terciária habilitada, as relações entre o número de habitantes e o número de unidades, o número de casos gerais de câncer e de câncer bucal estimados pelo INCA ${ }^{4}$ para 2018 e o número de unidades, o número de unidades com registro de cirurgia realizada para tratamento do câncer bucal e o número de cirurgias realizadas por unidade. Foram utilizadas informações da portaria de habilitação dessas unidades e do Sistema de Informação Hospitalar do SUS (disponíveis no período entre abril a setembro de 2017).

O referencial analítico utilizado foi a Teoria da Estruturação (TE) de Giddens ${ }^{17}$. A TE tem se apresentado como um recurso teórico para análise das políticas públicas de saúde, que são estruturadas com base na intencionalidade governamental e nas ações concretas de diversos atores na aplicabilidade dessas políticas. Possui potencial analítico e possibilita a utilização e integração de diversos métodos de investigação, pois não é definida por limites pré-estabelecidos ${ }^{18,19}$.

A TE se baseia na compreensão da "dualidade da estrutura", onde as propriedades estruturais do sistema social são compreendidas, ao mesmo tempo, como meio e resultado das práticas que elas recursivamente organizam ${ }^{17}$. Busca-se uma compreensão equilibrada entre a influência das estruturas sociais nos agentes e a liberdade de ação desses mesmos agentes para modificar essa estrutura ${ }^{20,21}$.

A estrutura é composta por um conjunto de regras e recursos. As regras correspondem aos procedimentos inscritos na consciência prática do ator, sendo operacionalizadas em suas ações. Incluem-se as prescrições formalizadas e as regras informais vivenciadas na integração social. Possuem aspecto normativo, referente aos direitos e obrigações, e aspecto semântico, que alude ao significado qualitativo e processual das práticas. Além disso, possuem papel regulador, que determina como algo deve ser feito para que não haja sanções ${ }^{17,19,21}$.

Os recursos, por sua vez, são as facilidades que o agente tem acesso para alcançar seus objetivos. São classificados como alocativos, que se referem ao controle de bens e objetos, e autoritativos, que são os recursos não materiais envolvidos na geração de poder. O poder é central na TE, sendo compreendido como a capacidade de controle de alguns atores pelos outros, o que depende da oportunidade e da relação existente entre os agentes $^{17,19}$.

Diante do objetivo desse estudo, foi realizada a análise classificada por Giddens como do "tipo institucional", enfatizando-se as propriedades estruturais do sistema de saúde por meio da análise das formas pelas quais a estrutura, por meio de regras (legislações, normas, protocolos) e recursos (humanos, financeiros, de estrutura física e autoritativos), tem moldado as práticas dos agentes envolvidos (gestores, profissionais e usuários) ${ }^{17}$.

Assim, os resultados foram organizados de forma a apresentar a estrutura na qual estão baseadas as práticas de controle do câncer bucal, considerando os diferentes componentes assistenciais previstos na PNPCC: Atenção Básica; Atenção Especializada Ambulatorial; Atenção Especializada Hospitalar; e Atenção Domiciliar ${ }^{22}$.

\section{Resultados e discussão}

Foram incluídas 10 (dez) normativas, publicadas entre 2004 e 2017 (seis são republicações em portarias de consolidação de 2017) (Quadro 1). Quatro se referem à organização das ações de saúde bucal ${ }^{22-24}$, cinco estão relacionadas à onco$\operatorname{logia^{25-27}}$ e uma ao processo de financiamento ${ }^{28}$.

A estruturação da atenção oncológica no SUS é realizada por meio da PNPCC de 2013, que aprimorou a Política Nacional de Atenção Oncológica (PNAO) de 2005. Seu objetivo é:

(...) a redução da mortalidade e da incapacidade causadas por esta doença e ainda a possibilidade de diminuir a incidência de alguns tipos de câncer, bem como contribuir para a melhoria da qualidade de vida dos usuários com câncer, por meio de ações de promoção, prevenção, detecção precoce, tratamento oportuno e cuidados paliativos ${ }^{22}$.

Na PNPCC são apresentados os aspectos normativos que definem as responsabilidades dos gestores das três esferas do SUS. Como responsabilidade comum, todos devem garantir os recursos alocativos necessários para organização da rede de atenção à saúde, como infraestrutura adequada, recursos humanos capacitados e qualificados, recursos materiais, equipamentos e insumos suficientes ${ }^{22}$.

Além disso, compete à esfera federal cooperar com os demais gestores na organização dos serviços, garantindo o financiamento, elaborando protocolos clínicos, definindo diretrizes para a organização das linhas de cuidado e monitorando o cumprimento do prazo de sessenta dias após o diagnóstico para início do tratamento ${ }^{22,25}$. Ao ente estadual compete definir estratégias de articulação com as direções municipais do SUS para a elaboração de planos e pactos regionais neces- 
Quadro 1. Normativas incluídas no estudo, por ano de publicação e objetivo.

\begin{tabular}{|c|c|c|}
\hline Ano & Normativa & Objetivo \\
\hline 2004 & $\begin{array}{l}\text { Diretrizes da Política Nacional } \\
\text { de Saúde Bucal }{ }^{23} \text {. }\end{array}$ & $\begin{array}{l}\text { Apresenta as diretrizes do Ministério da Saúde para a organização da } \\
\text { atenção à saúde bucal no âmbito do Sistema Único de Saúde. }\end{array}$ \\
\hline 2012 & $\begin{array}{l}\text { Lei no } 12.732 \text { de } 22 \text { de } \\
\text { novembro }^{25} \text {. }\end{array}$ & $\begin{array}{l}\text { Estabelece prazo para o início do tratamento de paciente com } \\
\text { neoplasia maligna comprovada. }\end{array}$ \\
\hline 2014 & $\begin{array}{l}\text { Portaria MS/SAS no } 140 \text { de } 27 \text { de } \\
\text { fevereiro }^{26} \text {. }\end{array}$ & $\begin{array}{l}\text { Redefine os critérios e parâmetros para organização, planejamento, } \\
\text { monitoramento, controle e avaliação dos estabelecimentos de saúde } \\
\text { habilitados na atenção especializada em oncologia e define as condições } \\
\text { estruturais, de funcionamento e de recursos humanos para a habilitação } \\
\text { destes estabelecimentos no âmbito do Sistema Único de Saúde. }\end{array}$ \\
\hline 2015 & $\begin{array}{l}\text { Portaria MS/SAS no } 516 \text { de } 17 \text { de } \\
\text { junho }{ }^{27} \text {. }\end{array}$ & $\begin{array}{l}\text { Aprova as Diretrizes Diagnósticas e Terapêuticas do Câncer de } \\
\text { Cabeça e Pescoço. }\end{array}$ \\
\hline 2017 & $\begin{array}{l}\text { Portaria de Consolidação MS/ } \\
\text { GM no } 2 \text { (Anexo IX) de } 03 \text { de } \\
\text { outubro }^{22} \text {. }\end{array}$ & $\begin{array}{l}\text { Instituí a Política Nacional para a Prevenção e Controle do Câncer } \\
\text { na Rede de Atenção à Saúde das Pessoas com Doenças Crônicas no } \\
\text { âmbito do Sistema Único de Saúde. }\end{array}$ \\
\hline 2017 & $\begin{array}{l}\text { Portaria de Consolidação MS/ } \\
\text { GM no } 2 \text { (Anexo XXII) de } 03 \text { de } \\
\text { outubro }^{22} \text {. }\end{array}$ & $\begin{array}{l}\text { Aprova a Política Nacional de Atenção Básica, com vistas à revisão } \\
\text { da regulamentação de implantação e operacionalização vigentes, no } \\
\text { âmbito do Sistema Único de Saúde, estabelecendo-se as diretrizes } \\
\text { para a organização do componente Atenção Básica, na Rede de } \\
\text { Atenção à Saúde. }\end{array}$ \\
\hline 2017 & $\begin{array}{l}\text { Portaria de Consolidação MS/ } \\
\text { GM no } 5 \text { (Seção I do Capítulo V } \\
\text { do Título IV) de } 03 \text { de outubro }{ }^{24} \text {. }\end{array}$ & $\begin{array}{l}\text { Instituí os Centros de Especialidades Odontológicas e de } \\
\text { Laboratórios Regionais de Próteses Dentárias, e estabelecidos os } \\
\text { critérios, normas e requisitos para seu credenciamento. }\end{array}$ \\
\hline 2017 & $\begin{array}{l}\text { Portaria de Consolidação MS/ } \\
\text { GM no } 5 \text { (Seção II do Capítulo I } \\
\text { do Título IV) de } 03 \text { de outubro }{ }^{24} \text {. }\end{array}$ & $\begin{array}{l}\text { Instituí o Programa Nacional de Melhoria do Acesso e da Qualidade } \\
\text { da Atenção Básica. }\end{array}$ \\
\hline 2017 & $\begin{array}{l}\text { Portaria de Consolidação no } \\
5 \text { (Seção II do Capítulo V do } \\
\text { Título IV) de } 03 \text { de outubro }{ }^{24} \text {. }\end{array}$ & $\begin{array}{l}\text { Dispõe sobre o Programa de Melhoria do Acesso e Qualidade dos } \\
\text { Centros de Especialidades Odontológicas. }\end{array}$ \\
\hline 2017 & $\begin{array}{l}\text { Portaria de Consolidação MS/ } \\
\text { GM n }{ }^{\circ} 6 \text { de } 03 \text { de outubro }{ }^{28} \text {. }\end{array}$ & $\begin{array}{l}\text { Consolida as normas sobre o financiamento e a transferência dos } \\
\text { recursos federais para as ações e os serviços de saúde do Sistema } \\
\text { Único de Saúde. }\end{array}$ \\
\hline
\end{tabular}

MS: Ministério da Saúde; SAS: Secretaria de Assistência à Saúde; GM: Gabinete do Ministro.

Fonte: Saúde Legis. Disponível em: http://portal2.saude.gov.br/saudelegis/LEG_NORMA_PESQ_CONSULTA.CFM.

sários para o controle do câncer. Além disso, cabe ao estado contratualizar os serviços que estão sob sua gestão e garantir e monitorar o cumprimento do prazo de sessenta dias ${ }^{22}$. Aos municípios compete pactuar regionalmente as ações e serviços necessários para a atenção integral da pessoa com câncer, além de contratualizar os serviços necessários quando não existir capacidade instalada no próprio município. Deve pactuar, também, a regulação e o fluxo de usuários, visando a garantia da referência e da contrarreferência ${ }^{22}$.

Ao apresentar as diferentes responsabilidades dos gestores, buscou-se regular as relações entre os entes federativos, principalmente na formulação dos pactos para configuração das redes regionalizadas. Entretanto, apesar da normatiza- ção, a distribuição dos recursos alocativos e autoritativos é bastante desigual, pois depende das oportunidades, do posicionamento e das relações existentes entre os agentes e suas instituições ${ }^{17}$. Isso faz com que os gestores e profissionais, a depender das relações de poder e do acesso aos recursos alocativos, podem ter maiores ou menores dificuldades em sua prática cotidiana do controle do câncer bucal.

\section{Atenção Básica}

O componente assistencial da atenção básica é regulado através da Política Nacional de Atenção Básica (PNAB). Desenvolve ações de saúde individuais, familiares e coletivas que abrangem 
promoção, prevenção, proteção, diagnóstico, tratamento, reabilitação, redução de danos, cuidados paliativos e vigilância em saúde. Trata-se da porta de entrada preferencial do sistema, responsável pela coordenação do cuidado e ordenação dos fluxos e contrafluxos dos usuários pela rede. Deve ser ofertada de forma universal, integral e gratuita $^{22}$.

A PNAB tem na ESF sua ação prioritária para expansão e qualificação desse nível de atenção ${ }^{22}$. A ESB integrada à equipe da ESF pode ser de Modalidade 1 (um cirurgião-dentista e um técnico de saúde bucal (TSB) e/ou auxiliar de saúde bucal (ASB)) ou Modalidade 2 (uma ESB da Modalidade 1 acrescida de um TSB). As ESB devem compartilhar a gestão e o processo de trabalho da equipe da ESF a que está vinculada, tendo responsabilidade sanitária pela mesma população e território adstrito ${ }^{22}$.

Cabe aos cirurgiões-dentistas da atenção básica a realização da atenção em saúde bucal (promoção e proteção da saúde, prevenção de agravos, diagnóstico, tratamento, acompanhamento, reabilitação e manutenção da saúde) individual e coletiva ${ }^{22}$. No âmbito da oncologia, a PNPCC estabelece para a atenção básica a função de realizar ações de promoção da saúde e prevenção de fatores de risco para o câncer. Dentre esses fatores, destaca-se para o câncer bucal a diminuição da prevalência do uso do tabaco e do álcool ${ }^{9,22}$.

Outra importante função da atenção básica destacada na PNPCC é o rastreamento de lesões precoces. Entretanto, para o câncer bucal não existem evidências científicas que o seu rastreamento resulta em aumento da sobrevida dos pacientes $^{29}$. Assim, cabe à ESB organizar outras estratégias, como o acompanhamento de grupos expostos frequentemente aos fatores de risco, visando a detecção precoce de lesões e posterior encaminhamento oportuno dos usuários para confirmação diagnóstica ${ }^{22,23}$.

A responsabilidade pelo financiamento da Atenção Básica é tripartite, mas, devido à instabilidade dos recursos estaduais e municipais, o gasto público em saúde no Brasil tem sido historicamente financiado por recursos federais ${ }^{30}$. Esse maior acesso aos recursos alocativos confere ao ministro da saúde o papel de agente determinador das políticas públicas, mesmo tratando-se de entes federativos independentes entre si. Porém, apesar de seu papel protagonista, a relação do ministro com os demais gestores não é isenta de conflitos, pois os secretários estaduais e municipais de saúde, mesmo possuindo menos recursos alocativos, possuem estratégias de articulação política que aumentam suas influências na determinação das políticas de saúde emanadas pelo Ministério da Saúde, o que ilustra a dialética de controle dessa relação.

O recurso federal desse componente é composto pelo Piso da Atenção Básica Fixo e pelo Piso da Atenção Básica Variável. Enquanto o primeiro é calculado por um valor per capita, o segundo é condicionado à implantação de estratégias e programas, servindo como importante fator indutor de implantação das ESB. Além do incentivo financeiro direto, o Plano de Fornecimento de Equipamentos Odontológicos para as ESB da ESF estabelece a doação, pelo Ministério da Saúde, de um equipo odontológico completo para cada equipe municipal implantada ${ }^{28}$.

Pela PNAB também foram estabelecidas medidas para ampliação do acesso de populações com vulnerabilidades específicas. A Unidade Odontológica Móvel (UOM), constituída de consultórios estruturados em veículos adaptados e equipados cedidos pelo Ministério da Saúde aos municípios, são destinadas para municípios com grande extensão territorial. Além da UOM, o município recebe um repasse mensal, devendo garantir materiais de consumo e profissionais ${ }^{22}$. As Equipes de Saúde da Família Ribeirinha (ESFR) e Equipes de Saúde da Família Fluvial (ESFF) são destinadas à Amazônia Legal e ao Pantanal Sul-Mato-Grossense e podem contar com a presença do cirurgião-dentista, com financiamento semelhante à ESB da $\mathrm{ESF}^{22,28}$. As equipes do Consultório na Rua (ECR) lidam com as diferentes necessidades de saúde da população em situação de rua e o cirurgião-dentista e o TSB podem fazer parte da equipe, não havendo diferenciação no repasse de recursos ${ }^{22,28}$. As equipes de saúde no Sistema Prisional (ESP), por sua vez, são constituídas por equipe multiprofissional e a presença do cirurgião-dentista e do TSB (ou ASB) é obrigatória. Há financiamento mensal que varia a depender do número de custodiados e modalidade das equipes ${ }^{22,28}$. Essas são importantes medidas de estruturação da atenção ao câncer bucal, haja vista que são destinadas as populações que possuem acesso limitado e são frequentemente expostas aos principais fatores de risco ${ }^{31-33}$.

O modelo de financiamento estimulou a expansão da ESF ao longo dos anos, incluindo as ESB (Tabela 1). Entre 2002 e 2017, o número de equipes da ESF aumentou em todas as regiões, destacando-se o Norte (2,69 vezes mais equipes). Em ritmo mais acelerado, o número de ESB aumentou em mais de cinco vezes no país, chegando a aumentar em nove vezes na região sudeste. 


\begin{tabular}{|c|c|c|c|c|c|c|c|}
\hline \multirow[t]{27}{*}{ 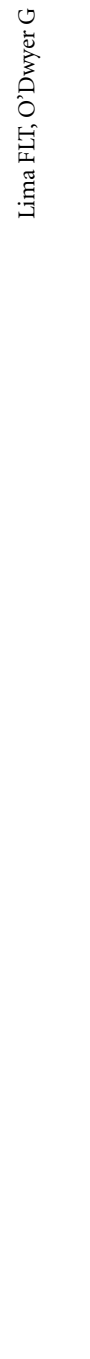 } & $\begin{array}{l}\text { Tabela 1. Variação } \\
\text { Bucal (ESB), cobe } \\
\text { brasileira. }\end{array}$ & $\begin{array}{l}\text { da população, ec } \\
\text { tura e municípic }\end{array}$ & $\begin{array}{l}\text { iipes da Estra } \\
\text { atendidos en }\end{array}$ & $\begin{array}{l}\text { ia de Saúde d } \\
002 \text { e 2017, er }\end{array}$ & $\begin{array}{l}\text { amília (ESF) } \\
\text { números }(\mathrm{N})\end{array}$ & $\begin{array}{l}\text { de Equipes d } \\
\text { percentual ( } \%\end{array}$ & $\begin{array}{l}\text { Saúde } \\
\text {, por região }\end{array}$ \\
\hline & & Centro-Oeste & Nordeste & Norte & Sudeste & Sul & Brasil \\
\hline & \multicolumn{7}{|l|}{ População (N) } \\
\hline & 2002 & 11.883 .997 & 48.328 .769 & 13.243 .229 & 73.469 .982 & 25.454 .344 & 172.358 .700 \\
\hline & 2017 & 15.660 .988 & 56.915 .936 & 17.740 .418 & 86.356 .952 & 29.439 .773 & 206.114 .067 \\
\hline & Variação & 1,32 & 1,18 & 1,34 & 1,18 & 1,16 & 1,20 \\
\hline & \multicolumn{7}{|l|}{ Equipes $(\mathrm{N})$} \\
\hline & ESF 2002 & 1.417 & 6.699 & 1.214 & 4.981 & 2.423 & 17.734 \\
\hline & ESF 2017 & 2.745 & 14.757 & 3.271 & 13.300 & 5.799 & 39.782 \\
\hline & Variação ESF & 1,94 & 2,20 & 2,69 & 2,67 & 2,39 & 2,24 \\
\hline & ESB 2002 & 534 & 2.134 & 242 & 705 & 464 & 4.261 \\
\hline & ESB 2017 & 1.933 & 10.700 & 1.812 & 6.486 & 3.122 & 24.053 \\
\hline & Variação ESB & 3,62 & 5,01 & 7,49 & 9,20 & 6,73 & 5,64 \\
\hline & \multicolumn{7}{|l|}{ Cobertura (\%) } \\
\hline & ESF 2002 & 38,97 & 45,33 & 30,56 & 22,63 & 30,73 & 31,93 \\
\hline & ESF 2017 & 55,70 & 75,13 & 57,97 & 49,93 & 61,95 & 59,74 \\
\hline & Variação ESF & 1,43 & 1,66 & 1,90 & 2,21 & 2,02 & 1,87 \\
\hline & ESB 2002 & 26,00 & 28,00 & 11,00 & 6,00 & 14,00 & 15,00 \\
\hline & ESB 2017 & 39,94 & 56,78 & 33,26 & 24,47 & 34,33 & 36,73 \\
\hline & Variação ESB & 1,54 & 2,03 & 3,02 & 4,08 & 2,45 & 2,45 \\
\hline & \multicolumn{7}{|l|}{ Municípios (N) } \\
\hline & ESF 2002 & 439 & 1.408 & 328 & 1.171 & 817 & 4.163 \\
\hline & ESF 2017 & 458 & 1.786 & 445 & 1.574 & 1.139 & 5.402 \\
\hline & Variação ESF & 1,04 & 1,27 & 1,36 & 1,34 & 1,39 & 1,30 \\
\hline & ESB 2002 & 349 & 1.025 & 154 & 334 & 440 & 2.302 \\
\hline & ESB 2017 & 448 & 1.755 & 420 & 1.329 & 981 & 4.933 \\
\hline & Variação ESB & 1,28 & 1,71 & 2,73 & 3,98 & 2,23 & 2,14 \\
\hline
\end{tabular}

Fonte: Sala de Apoio à Gestão Estratégica do Ministério da Saúde (SAGE). Disponível em: http://sage.saude.gov.br/.

Acompanhando esses números, a cobertura dos serviços da ESF e das ESB aumentou em todo o país $(1,87$ e 2,45 vezes maior, respectivamente). Já o número de municípios atendidos pela ESF aumentou cerca de 30\%, alcançando cerca de $97 \%$ dos municípios brasileiros. Já as ESB dobraram em quantidade de municípios cobertos, atingindo 88,5\% dos municípios em 2017.

Observa-se que a expansão das ESB foi em ritmo mais intenso do que da ESF durante o período estudado. Esse resultado está associado a três fatores inter-relacionados: a escassez de serviços de saúde bucal no período anterior à inserção da ESB na ESF, o atraso da inclusão da ESB na ESF e o processo de estruturação proporcionado pela PNSB e PNAB.

A inclusão da ESB na ESF buscou abranger uma demanda historicamente reprimida, sendo um importante passo na reorganização da aten- ção à saúde bucal, viabilizando a expansão da atenção básica de forma universal e rompendo com as políticas centradas em grupos restritos ${ }^{34}$. Essa inclusão, realizada em 2000, seguiu uma proporção de duas equipes da ESF para cada ESB. Apenas em 2003, com a estruturação da PNSB e a priorização das ações da atenção básica, foi possibilitada a ampliação do número de ESB até se igualarem às equipes da ESF. Assim, o maior ritmo de implantação das ESB identificado neste estudo ocorreu quando o processo de implantação das equipes de ESF já estava estabilizado.

Apesar da expressiva expansão das ESB observada, a atual cobertura de $36,73 \%$ é bastante limitada, considerando que se trata de um serviço de atenção básica inserido em um sistema de saúde universal ${ }^{35}$. A baixa cobertura, associada com a sobrecarga de trabalho das ESB, faz com que um grande contingente populacional fique 
com acesso limitado (ou sem acesso) às ações de promoção da saúde, prevenção e diagnóstico do câncer bucal. Assim, apesar do aumento do investimento público, este ainda é insuficiente frente as necessidades acumuladas e a crescente demanda de serviços pela população ${ }^{35}$.

Além da ampliação de serviços, é necessário observar a qualidade em que estes estão sendo prestados. O Programa Nacional de Melhoria do Acesso e da Qualidade da Atenção Básica (PMAQ-AB) foi lançado em 2011 e se encontra no terceiro ciclo $^{24}$. A adesão ao programa é voluntária, mas tem sido fortemente incentivada por repasses financeiros federais ${ }^{19,23}$. Mais do que um novo instrumento de financiamento e expressão de poder do ente federal, o PMAQ-AB deve servir como importante ferramenta de diagnóstico da qualidade dos serviços.

Dados do primeiro ciclo mostram que $72,66 \%$ das ESB afirmam fazer campanhas para detecção de lesões suspeitas de malignidade e encaminhamento dos casos. Apesar dessa alta porcentagem, é preciso destacar que não há informações sobre o tipo e método da campanha realizada, a frequência de realização, o público alcançado e, principalmente, a efetividade das mesmas na detecção precoce do câncer bucal ou no aumento de sobrevida dos usuários com lesão identificada. Destaca-se ainda que 48,3\% relatam esperar 365 dias ou mais para conseguir consulta com o profissional especialista, 40,9\% não registra e acompanha os casos suspeitos e confirmados e apenas $45,8 \%$ relataram existir fluxos preferenciais para usuários com suspeita de câncer bucal $^{36}$. Ou seja, apesar da normatização da atenção básica, poucas são as mudanças das práticas assistenciais direcionadas à essa enfermidade.

Diante desse cenário, observa-se que as regras definidas através das políticas de oncologia e de saúde bucal convergem sobre o papel da atenção básica no controle do câncer bucal. Essas políticas apresentam aspectos facilitadores do controle dessa neoplasia, como a incorporação da ESB nas diversas estratégias de conformação de equipes na atenção básica (ESF, ESFR, ESFF, ECR e ESP) e o aumento do financiamento federal para implantação e custeio dessas equipes. Porém, apresentam também aspectos coercitivos, impostos aos profissionais das ESB, como a baixa cobertura assistencial e a sobrecarga de trabalho, o que dificulta o diagnóstico e tratamento precoce do câncer bucal.

\section{Atenção Especializada Ambulatorial}

A Atenção Especializada Ambulatorial prevista na PNPCC é composta por serviços do segundo nível de atenção, capazes de realizar assistência diagnóstica e terapêutica, além de assegurar o encaminhamento dos usuários com suspeição ou confirmação diagnóstica de câncer para as unidades hospitalares ${ }^{22}$.

Na PNSB o CEO é o responsável em prestar esses serviços. Há financiamento federal específico para implantação e custeio do CEO, a depender da modalidade do serviço: CEO Tipo I (3 consultórios), CEO Tipo II (4 a 6 consultórios), CEO Tipo III (7 ou mais consultórios) ${ }^{28}$. Os CEO assumem papel estratégico na detecção do câncer bucal no SUS, pois todas as unidades devem realizar, entre outros serviços, o diagnóstico e detecção do câncer bucal ${ }^{24}$.

De forma geral, o CEO tem sido considerado um avanço, pois representa uma ruptura com a histórica limitação de oferta a cuidados especializados em saúde bucal ${ }^{37}$. Conforme observa-se na Tabela 2, o incentivo dado através da PNSB fez com que o número de CEO aumentasse ao longo dos anos, alcançado 1.033 estabelecimentos em 2016 (valor dez vezes maior do que em 2004). A região nordeste foi a que teve o maior acréscimo de unidades (16 vezes). A relação entre população e o número de unidades diminuiu oito vezes no Brasil, chegando à média de cerca de 200.000 habitantes por CEO. Em 2016, o Nordeste alcançou a melhor relação entre população e número de CEO (141.427 habitantes por CEO) e o Norte a pior relação (260.410 habitantes por CEO). O número de municípios com unidades instaladas aumentou em 14 vezes no país, alcançando 857 municípios. Apesar do Sudeste ser a região onde houve o maior aumento proporcional do número de municípios (22,46 vezes), o Nordeste é a região que possui o maior número de municípios atendidos (356 municípios).

Verifica-se que a PNSB estimulou a expansão de serviços especializados em odontologia (dentre eles o diagnóstico do câncer bucal) por todas as regiões do país. Entretanto, a expansão é limitada a poucos municípios (15\% das cidades), o que resulta em dificuldade de acesso para uma parcela da população. Além disso, o modelo de expansão aumentou a desigualdade de acesso, pois o direcionamento de recursos foi feito para cidades que já apresentavam os melhores indicadores sociais ${ }^{38}$. Frente ao processo incipiente de regionalização do cuidado bucal ${ }^{39}$, é importante investir de forma mais enérgica no CEO como 


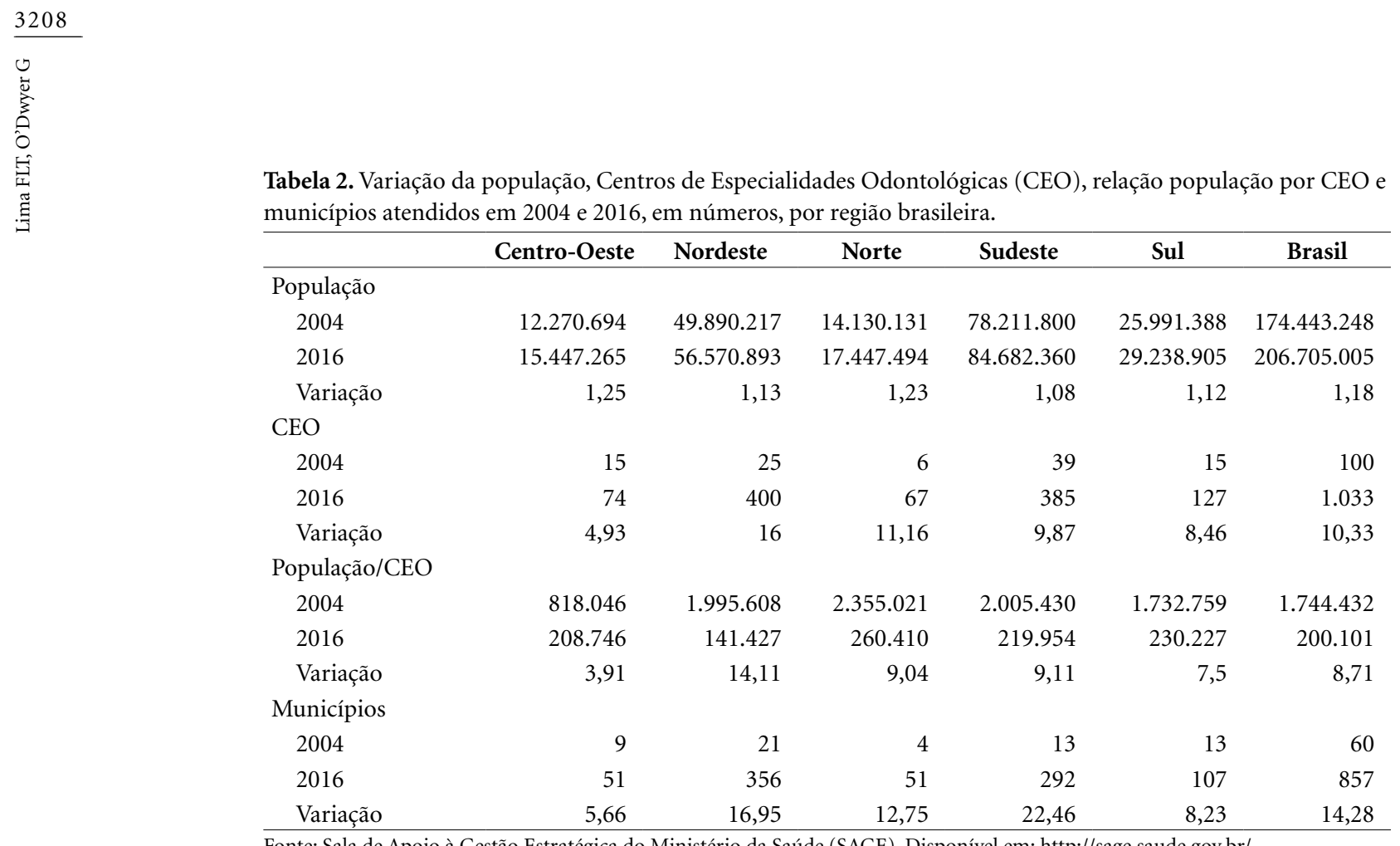

Fonte: Sala de Apoio à Gestão Estratégica do Ministério da Saúde (SAGE). Disponível em: http://sage.saude.gov.br/.

um estabelecimento de âmbito regional (conforme já preconiza a legislação), para que os moradores de pequenos municípios também acessem esses serviços.

Outra estratégia para expansão do diagnóstico do câncer bucal que tem sido discutida é o estímulo para que profissionais da atenção básica também possam realizar as biópsias ${ }^{8}$. Porém, sabe-se que esses cirurgiões-dentistas, além de sobrecarregados com outras demandas, não se sentem habilitados para realizar esse procedimento $^{40}$, sendo necessário investimento em educação permanente e em materiais e instrumentais necessários.

Os repasses financeiros do CEO são vinculados a metas de produção para cada especialidade e modalidade de implantação. Porém, não há uma meta específica para o serviço de diagnóstico bucal, estando as biópsias de lesões suspeitas inseridas no grupo de procedimentos de cirurgia oral $^{28}$. A ausência de uma meta específica pode fazer com que os gestores direcionem os serviços para outras prioridades e não se sintam obrigados a oferecer esse serviço. Um indicativo dessa situação foi apresentado em estudo realizado com dados do PMAQ-AB referentes ao estado do Rio de Janeiro. Entre as ESB que possuíam um CEO de referência, $25 \%$ responderam negativamente para acesso à especialidade de estomato- logia. Ou seja, mesmo sendo essa a especialidade odontológica mais habilitada para o diagnóstico do câncer bucal e esse serviço ser obrigatório nos CEO, um número significativo de ESB está com dificuldades para confirmação diagnóstica das lesões suspeitas ${ }^{41}$.

Visando qualificar as ações, foi criado em 2013 o Programa de Melhoria do Acesso e Qualidade dos Centros de Especialidades Odontológicas (PMAQ-CEO), que trouxe a definição de parâmetros de qualidade para essas unidades ${ }^{25}$. Assim como no PMAQ-AB, o incentivo financeiro tem servido como incentivo para adesão dos municípios ${ }^{28}$. Ainda não foram encontrados estudos referentes ao primeiro ciclo do PMAQCEO. Esse tipo de ação é imprescindível, pois sabe-se do descompasso existente entre a proposta da política e a prática no cotidiano dos serviços ${ }^{42}$.

\section{Atenção Especializada Hospitalar}

A Atenção Especializada Hospitalar em oncologia é composta pelas unidades habilitadas como Centros de Assistência de Alta Complexidade em Oncologia (Cacon), Unidades de Assistência de Alta Complexidade em Oncologia (Unacon) e pelos Hospitais Gerais com Cirurgia Oncológica. Esses serviços têm o objetivo de oferecer tratamentos especializados de alta com- 
plexidade para as pessoas com câncer, ofertar e orientar os cuidados paliativos na internação e na assistência ambulatorial e domiciliar ${ }^{22}$.

Os Cacon são estabelecimentos que realizam diagnóstico definitivo e tratamento de todos os tipos de câncer (mas não obrigatoriamente para os cânceres raros e infantis). Todos Cacon devem oferecer cirurgia, radioterapia e quimioterapia dentro de sua estrutura hospitalar. Os Unacon realizam o diagnóstico definitivo e o tratamento apenas para os cânceres mais prevalentes, oferecendo minimamente os tratamentos cirúrgico e quimioterápico. Caso não ofereçam tratamento radioterápico, devem contratualizar formalmente esse serviço com outra unidade. Os Hospitais Gerais com Cirurgia Oncológica procedem o tratamento cirúrgico do câncer e realizam o encaminhamento, de forma regulada, dos casos que necessitam de complementação terapêutica ${ }^{22}$.

Na Portaria MS/SAS no 140 de 2014 são apresentados os critérios e parâmetros para organização, planejamento, monitoramento, controle e avaliação dos estabelecimentos habilitados na atenção especializada em oncologia ${ }^{26}$. Esse aspecto é denominado por Giddens como regulador, pois define os limites de atuação que cada estrutura possui ${ }^{17}$.

Segundo essa Portaria, o processo de habilitação deve obedecer a razão de um estabelecimento para cada 500.000 habitantes. É autorizado à região Norte a habilitação de um Unacon em regiões com menos que 500.000 habitantes e baixa densidade demográfica. Por sua vez, nas regiões Sul e Sudeste é admitida habilitação de Cacon ou Unacon em áreas com menos que 500.000 habitantes, desde que haja estimativa de 900 casos novos anuais de câncer ${ }^{26}$.

Na Tabela 3 é observado o número de estabelecimentos habilitados na atenção hospitalar especializada em oncologia em 2017 por região brasileira $^{43}$, destacando-se que $48 \%$ dos serviços estão no Sudeste. Essa concentração de serviços está associada a dados demográficos (maior densidade demográfica e população mais idosa), epidemiológicos (maior incidência de câncer) e econômicos (região com municípios mais recursos financeiros). Verifica-se que, apesar das diferenças regionais existentes na relação do número de habitantes por unidade habilitada em oncologia, o número de casos de câncer e de câncer bucal estimados para 2018 por unidade especializada é bastante semelhante entre as regiões (Tabela 3 ).

$\mathrm{O}$ tratamento do câncer bucal deve seguir as Diretrizes Diagnósticas e Terapêuticas do Câncer de Cabeça e Pescoço, publicadas pelo Ministério da Saúde em 2015. Nesse documento são definidos os critérios de diagnóstico, tratamento e mecanismos de regulação, controle e avaliação de caráter nacional. Segundo essas diretrizes, os usuários devem ser preferencialmente atendidos em hospitais habilitados como Cacon ou Unacon com radioterapia ${ }^{27}$.

De fato, todos os Cacon devem realizar tratamento para o câncer bucal. O tratamento nas Unacon e nos Hospitais Gerais de Cirurgia Oncológica pode ser realizado, desde que haja programação para esse serviço definido pelas comissões intergestores do SUS ${ }^{26}$. No período de seis meses após a publicação da portaria de habilitação, 219 estabelecimentos, dos 299 habilitados, lançaram no Sistema de Informação Hospitalar do SUS a realização de 1.891 cirurgias para tratamento do câncer bucal (Tabela 4).

É possível observar que $73 \%$ das unidades habilitadas no país realizaram cirurgia para o câncer bucal no período analisado. Dois Cacon não lançaram a realização do procedimento, que deve ser obrigatório nesses serviços ${ }^{26}$. Verifica-se que a maioria das cirurgias foram realizadas nas Unacon, haja vista o maior número dessas unidades. Porém, os Cacon possuem produção média três vezes maior que as Unacon. Ao olharmos as diferenças regionais, verificamos novamente as desigualdades de acesso, onde a região Norte possui o menor número de unidades habilitadas, bem como o menor número de cirurgias realizadas, enquanto o Sudeste possui os indicadores com valores mais elevados.

As regras de implantação e funcionamento dos Cacon e Unacon são importantes instrumentos normativos de estruturação dos serviços especializados em oncologia. Essas normas apresentam requisitos mínimos que buscam garantir a qualidade dos serviços prestados à população. Entretanto, apesar desse aspecto facilitador para padronização e qualidade, essas normas podem dificultar a implantação de serviços nas regiões onde os gestores têm a sua disposição menos recursos alocativos. Essa situação se agrava ao observarmos que não foi estabelecida uma política de financiamento específica ${ }^{44}$, conforme ocorreu nos outros níveis de atenção.

\section{Atenção Domiciliar}

A atenção domiciliar, no âmbito da PNPCC, se refere aos cuidados paliativos dos usuários com câncer, que devem ser realizados de forma compartilhada com as equipes de atenção básica e articulados com as unidades especializadas. As 
Tabela 3. Distribuição das unidades habilitadas em oncologia e da relação entre o número de habitantes, casos estimados de câncer e casos estimados de câncer bucal, por unidade e região brasileira, 2018.

\begin{tabular}{lrrrrrr}
\hline \multicolumn{1}{c}{ Unidades } & $\begin{array}{c}\text { Centro- } \\
\text { Oeste }\end{array}$ & Nordeste & Norte & Sudeste & Sul & Brasil \\
\hline Cacon & 2 & 10 & 1 & 22 & 9 & 44 \\
Hospital Geral com Cirurgia & 0 & 0 & 1 & 7 & 0 & 8 \\
Oncológica & & & & & & \\
Unacon & 19 & 47 & 9 & 113 & 58 & 246 \\
Total & & & & & & \\
Unidades habilitadas & $21(7 \%)$ & $57(19 \%)$ & $11(3 \%)$ & $142(48 \%)$ & $67(23 \%)$ & 298 \\
Habitantes por unidade & 755.996 & 1.004 .459 & 1.630 .564 & 612.322 & 442.462 & 696.849 \\
Casos de câncer por unidade & 2.077 & 2.058 & 2.124 & 1.917 & 1.877 & 1.954 \\
Casos de câncer bucal por unidade & 49 & 49 & 45 & 53 & 42 & 49 \\
\hline
\end{tabular}

Cacon: Centros de Assistência de Alta Complexidade em Oncologia; Unacon: Unidades de Assistência de Alta Complexidade em Oncologia. ${ }^{\star}$ Casos estimados para 2018.

Fontes: INCA, 2017³; Portaria MS/SAS no 458 de 2017³; Sala de Apoio à Gestão Estratégica do Ministério da Saúde. Disponível em http://sage.saude.gov.br/\#.

Tabela 4. Número de hospitais habilitados em Oncologia que lançaram no Sistema de Informação Hospitalar do SUS a realização de cirurgia para tratamento do câncer bucal e número dessas cirurgias no período entre abril a setembro de 2017, por região brasileira.

\begin{tabular}{|c|c|c|c|c|c|c|}
\hline Unidades & $\begin{array}{c}\text { Centro- } \\
\text { Oeste }\end{array}$ & Nordeste & Norte & Sudeste & Sul & Brasil \\
\hline \multicolumn{7}{|l|}{ Cacon } \\
\hline Unidades & 2 & 10 & 1 & 22 & 9 & 44 \\
\hline Unidades com registro de cirurgia & 2 & 9 & 1 & 21 & 9 & 42 \\
\hline$\%$ de unidades com registro de cirurgia & 100 & 90 & 100 & 95 & 100 & 95 \\
\hline Número de cirurgias & 88 & 148 & 4 & 389 & 133 & 762 \\
\hline Número de cirurgias por unidade & 44,0 & 16,4 & 4,0 & 18,5 & 14,7 & 18,14 \\
\hline \multicolumn{7}{|l|}{ Hospital Geral com Cirurgia Oncológica } \\
\hline Unidades & 0 & 0 & 1 & 7 & 0 & 8 \\
\hline Unidades com registro de cirurgia & 0 & 0 & 0 & 2 & 0 & 2 \\
\hline$\%$ de unidades com registro de cirurgia & 0 & 0 & 0 & 28 & 0 & 25 \\
\hline Número de cirurgias & 0 & 0 & 0 & 3 & 0 & 3 \\
\hline Número de cirurgias por unidade & 0 & 0 & 0 & 1,5 & 0 & 1,5 \\
\hline \multicolumn{7}{|l|}{ Unacon } \\
\hline Unidades & 19 & 47 & 9 & 113 & 58 & 246 \\
\hline Unidades com registro de cirurgia & 13 & 25 & 8 & 79 & 50 & 175 \\
\hline$\%$ de unidades com registro de cirurgia & 68 & 53 & 89 & 70 & 86 & 71 \\
\hline Número de cirurgias & 50 & 233 & 54 & 509 & 280 & 1.126 \\
\hline Número de cirurgias por unidade & 3,8 & 9,3 & 6,8 & 6,4 & 5,6 & 6,4 \\
\hline \multicolumn{7}{|l|}{ Total } \\
\hline Unidades & 21 & 57 & 11 & 142 & 67 & 298 \\
\hline Unidades com registro de cirurgia & 15 & 34 & 9 & 102 & 59 & 219 \\
\hline$\%$ de unidades com registro de cirurgia & 71 & 59 & 81 & 72 & 88 & 73 \\
\hline Número de cirurgias & 138 & 381 & 58 & 901 & 413 & 1.891 \\
\hline Número de cirurgias por unidade & 9,2 & 11,2 & 6,4 & 8,8 & 7,0 & 8,6 \\
\hline
\end{tabular}

Cacon: Centros de Assistência de Alta Complexidade em Oncologia; Unacon: Unidades de Assistência de Alta Complexidade em Oncologia.

Fontes: Portaria MS/SAS no 458 de $2017^{43}$; Sistema de Informação Hospitalar do SUS. Disponível em: http://sihd.datasus.gov.br/ principal/index.php. 
Equipes Multiprofissionais de Atenção Domiciliar (EMAD) devem respeitar a cultura e os valores das famílias, dando ênfase no controle dos sintomas e na comunicação clara com os usuários e familiares ${ }^{22}$.

Essa modalidade de cuidado tem ganhado destaque não apenas no Brasil. Em um estudo com 1.290 pacientes com câncer bucal acompanhados por 20 anos na Inglaterra, identificou-se que os pacientes estão mais propensos a morrer em casa ou em uma unidade especializada em cuidados paliativos do que no passado. Segundo os autores, isso deve ser visto como uma tendência positiva, haja vista que corresponde a preferência dos pacientes ${ }^{45}$.

No Brasil, as EMAD são compostas por mé$\operatorname{dico}(\mathrm{s})$, enfermeiro(s), fisioterapeuta(s) ou assistente(s) social(is) e técnico(s) ou auxiliar(es) de enfermagem. A essa equipe pode-se integrar uma Equipe Multiprofissional de Apoio (EMAP), que pode ser composta por diferentes profissionais, entre eles o cirurgião-dentista. A PNSB não aborda essa modalidade de cuidado ${ }^{23,24}$.

As normas referentes ao cuidado domiciliar têm viabilizado o desenvolvimento de importantes serviços que visam a melhoria da qualidade de vida dos usuários sem possibilidades de cura. Entretanto, há uma limitação prevista na norma que inviabiliza a implantação na maioria dos municípios brasileiros, pois é necessário que o município tenha mais que 20.000 habitantes, possua um hospital de referência e seja coberto por um Serviço de Atendimento Móvel de Urgência (SAMU $192)^{24}$. Frente à essa limitação imposta pela regra, gestores e profissionais de pequenos municípios devem desenvolver estratégias para que os usuários não fiquem desamparados. O fortalecimento da rede de atenção, integrando a atenção básica local com os serviços especializados regionais, bem como a instrumentalização de cuidadores e familiares para o cuidado paliativo domiciliar, são alguns dos caminhos possíveis ${ }^{24}$.

Destacamos que a principal limitação do estudo é que não houve acesso à prática assistencial, focando-se nas propriedades estruturais do sistema de saúde, conforme orienta a pesquisa do tipo institucional de Giddens. Cabe a estudos futuros a realização da análise da "conduta estratégica", que foca sobre os modos como os agentes se apoiam nas propriedades estruturais para a constituição das ações, dando-se primazia à análise das consciências discursivas e práticas dos agentes.

\section{Considerações finais}

Há convergência entre a PNPCC e a PNSB na organização das ações direcionadas à prevenção e controle do câncer bucal. A preocupação governamental com os processos regulatórios dos recursos autoritativos e com o aumento na destinação de recursos alocativos direcionou a expansão dos serviços nos últimos 15 anos em todo o país. Porém, apesar da expansão, a cobertura populacional continua baixa, o que dificulta o diagnóstico e tratamento em tempo hábil e reflete diretamente na qualidade e tempo de sobrevida dos usuários, bem como no aumento dos custos para o sistema.

É necessário investir no processo de regionalização e universalização dos serviços para diminuir as desigualdades de acesso. Porém, em tempos de austeridade fiscal e cortes de gastos em programas sociais, o cenário torna-se preocupante. Um possível retrocesso nessas políticas pode agravar a situação e atingir aqueles que mais necessitam do SUS, contribuindo para o aumento dessas desigualdades. 


\section{Colaboradores}

FLT Lima participou na concepção, delineamento do estudo, coleta e análise das informações e redação do artigo. G O'Dwyer participou no delineamento do estudo, na análise das informações e na revisão final do artigo.

\section{Referências}

1. Biazevic MGH, Antunes JLF. Câncer Bucal. In: Antunes JLFA, Peres MA. Epidemiologia da Saúde Bucal. Rio de Janeiro: Guanabara Koogan, 2006.

2. Tapia JL, Goldberg LJ. The challenges of defining oral cancer: analysis of an ontological approach. Head and Neck Pathol 2011; 5(4):376-384.

3. Instituto Nacional de Câncer José Alencar Gomes da Silva (INCA). Informativo Deteç̧ão Precoce. Boletim ano $7, n^{\circ}$ 1. Rio de Janeiro: INCA; 2016.

4. Instituto Nacional de Câncer José Alencar Gomes da Silva (INCA). Estimativa 2018: incidência de câncer no Brasil. Rio de Janeiro: INCA; 2017.

5. Dias AA, Sampaio JJC, Rego DM, Lima DLF, Dalcico R. Políticas públicas e epidemiologia do câncer de boca. In: Dias AA. Saúde Bucal Coletiva: metodologia de trabalho e práticas. São Paulo: Santos; 2006. p. 297 314 .

6. Torre LA, Bray F, Siegel RL, Ferlay J, Lortet Tieulent J, Jemal A. Global cancer statistics, 2012. CA Cancer J Clin 2015; 65(2):87-108.

7. Montoro JRMC, Hicz HÁ, Souza L, Livingstone D, Melo DH, Tiveron RC, et al. Fatores prognósticos no carcinoma espinocelular de cavidade oral. Rev Bras Otorrinolaringol 2008; 74(6):861-866.

8. Torres-Perreira CC, Angelim-Dias A, Melo NS, Lemos-Jr CA, Oliveira EMF. Abordagem do câncer da boca: uma estratégia para os níveis primário e secundário de atenção em saúde. Cad Saude Publica 2012; 28(Supl. 1):30-39.

9. Bonfant GMS, Machado CJ, Souza PEA, Andrade ELG, Acurcio FA, Cherchiglia ML. Sobrevida de cinco anos e fatores associados ao câncer de boca para pacientes em tratamento oncológico ambulatorial pelo Sistema Único de Saúde, Brasil. Cad Saude Publica 2014; 30(5):983-997.

10. Visentin A, Lenardt MH. O itinerário terapêutico: história oral de idosos com câncer. Acta Paul Enferm 2010; 23(4):486-492.

11. Almeida FCS, Cazal C, Pucca Júnior GA, Silva DP, Frias AC, Araújo ME. Reorganization of Secondary and Tertiary Health Care Levels: Impact on the Outcomes of Oral Cancer Screening in the São Paulo State. Braz Dent J 2012; 23(3):241-255.

12. Cunha AR, Bavaresco CS, Carrard VC, Lombardo EM. Atrasos nos encaminhamentos de pacientes com suspeita de câncer bucal: percepção dos cirurgiõesdentistas na atenção básica à saúde. J Bras Tele 2013; 2(2):66-74.

13. Lombardo EM, Cunha AR, Carrard VC, Bavaresco CS. Atrasos nos encaminhamentos de pacientes com câncer bucal: avaliação qualitativa da percepção dos cirurgiões-dentistas. Cien Saude Colet 2014; 19(4):1223-1232.

14. Saltz E. Projeto de Expansão da Prevenção e Controle do Câncer de Boca: quinquenio 1988-93. R Bras Canc 1988; 34(4):221-239.

15. Teles JCB. Assistência odontológica no país: perspectivas 1. Cad Saúde Púbica 1985; 1(2):253-262.

16. Chaves SCL, Almeida AMFL, Rossi TRA, Santana SF, Barros SG, Santos CML. Política de Saúde Bucal no Brasil 2003-2014: cenário, propostas, ações e resultados. Cien Saude Colet 2017; 22(6):1791-1803. 
17. Giddens A. A constituição da sociedade. São Paulo: Martins Fontes; 2003.

18. O’Dwyer G, Mattos RA. Teoria da Estruturação de Giddens e os estudos de práticas avaliativas. Physis 2010; 20(2):609-623.

19. O’Dwyer G. Estudos de políticas e a Teoria da Estruturação de Giddens. In: Baptista TWF, Azevedo CS, Machado CV. Politicas, Planejamento e Gestão em Saúde: abordagens e métodos de pesquisa. Rio de Janeiro: Editora Fiocruz; 2015. p. 173-192.

20. Giddens A, Sutton PW. Conceitos essenciais da Sociologia. São Paulo: Editora Unesp; 2016.

21. Silva FRR. O caminho da constituição da sociedade: a teoria da estruturação de Anthony Giddens. Jundiaí: Paco Editorial; 2016.

22. Brasil. Portaria de Consolidação $n^{\circ} 2$, de 28 de setembro de 2017. Consolidação das normas sobre as políticas nacionais de saúde do Sistema Único de Saúde. Diário Oficial da União 2017; 03 out.

23. Brasil. Ministério da Saúde (MS). Secretaria de Atenção à Saúde. Departamento de Atenção Básica. Diretrizes da Política Nacional de Saúde Bucal. Brasília: MS; 2004.

24. Brasil. Portaria de Consolidação no 5 , de 28 de setembro de 2017. Consolidação das normas sobre as políticas nacionais de saúde do Sistema Único de Saúde. Diário Oficial da União 2017; 03 out.

25. Brasil. Lei no 12.732, de 22 de novembro de 2012. Dispõe sobre o primeiro tratamento de paciente com neoplasia maligna comprovada e estabelece prazo para seu início. Diário Oficial da União 2012; 23 nov.

26. Brasil. Portaria $\mathrm{n}^{\circ} 140$, de 27 de fevereiro de 2014 Redefine os critérios e parâmetros para organização, planejamento, monitoramento, controle e avaliação dos estabelecimentos de saúde habilitados na atenção especializada em oncologia e define as condições estruturais, de funcionamento e de recursos humanos para a habilitação destes estabelecimentos no âmbito do SUS. Diário Oficial da União 2014; 27 fev.

27. Brasil. Portaria no 516 , de 17 de junho de 2015. Aprova as Diretrizes Diagnósticas e Terapêuticas do Câncer de Cabeça e Pescoço. Diário Oficial da União 2015; 18 jun.

28. Brasil. Portaria de Consolidação no 6 , de 28 de setembro de 2017. Consolidação das normas sobre o financiamento e a transferência dos recursos federais para as ações e os serviços de saúde do Sistema Único de Saúde. Diário Oficial da União 2017; 30 out.

29. Rethman MP, Carpenter W, Cohen EE, Epstein J, Evans CA, Flaitz CM, et al. American Dental Association Council on Scientific Affairs Expert Panel on Screening for Oral Souamous Cell Carcinomas. Evidence-based clinical recommendations regarding screening for oral squamous cell carcinomas. Tex Dent J 2012; 129(5):491-507.

30. Mendes A, Marques RMM. O financiamento da Atenção Básica e da Estratégia Saúde da Família no Sistema Único de Saúde. Saude Debate 2014; 38(103):900-916.

31. World Health Organization (WHO). Health in prisons: a WHO guide to the essentials in prison health. Copenhagen: WHO; 2007.
32. Pukkala E, Martinsen JI, Lynge E, Gunnarsdottir HK Sparén P, Tryggvadottir L, Weiderpass E, Kjaerheim K. Occupation and cancer - follow-up of 15 million people in five Nordic countries. Acta Oncol 2009; 48(5):646-790.

33. Van Hout MC, Hearne E. Oral health behaviours amongst homeless people attending rehabilitation services in Ireland. J Ir Den Assoc 2014; 60(3):144-149.

34. Gigante EC, Guimarães JP. A trajetória da saúde bucal pelas políticas públicas no Brasil a partir da criação do SUS. Cad Saude Desenv 2013; 3(2):66-77.

35. Nascimento AC, Moysés ST, Werneck RI, Moysés SJ. Oral health in the context of primary care in Brazil. Int Dent J 2013; 63(5):237-243.

36. Casotti E, Contarato PC, Fonseca ABM, Borges PKO, Baldani MH. Atenção em Saúde Bucal no Brasil: uma análise a partir da Avaliação Externa do PMAQ-AB. Saude Debate 2014; 38(esp):140-157.

37. Frazão P, Narvai PC. Saúde bucal no Sistema Único de Saúde: 20 anos de lutas por uma política pública. Saude Debate 2009; 33(81):64-71.

38. Lino PA, Werneck MAF, Lucas SD, Abreu MHNG. Análise da atenção secundaria em saúde bucal no estado de Minas Gerais. Cien Saude Colet 2014; 19(9):3879-3888.

39. Mello ALSF, Andrade SR, Moysés SJ, Erdmann, AL. Saúde bucal na rede de atenção e processo de regionalização. Cien Saude Colet 2014; 19(1):205-214.

40. Noro LRA, Landim JR, Martins MCA, Lima YCP. The challenge of the approach to oral cancer in primary health care. Cien Saude Colet 2017; 22(5):1579-1587.

41. Casotti E, Monteiro ABF, Castro-Filho EL, Santos MP. Organização dos serviços públicos de saúde bucal para diagnóstico precoce de desordens com potencial de malignização do estado do Rio de Janeiro, Brasil. Cien Saude Colet 2014; 21(5):1573-1582.

42. Pires FS, Botazzo C. Organização tecnológica do trabalho em saúde bucal no SUS: uma arqueologia da política nacional de saúde bucal. Saude Soc 2015; 24(1):273-284.

43. Brasil. Portaria $n^{\circ} 458$, de 24 de fevereiro de 2017. Mantem as habilitações de estabelecimentos de saúde na Alta Complexidade e exclui prazo estabelecido na Portaria no 140/SAS/MS, de 27 de fevereiro de 2014. Diário Oficial da União 2017; 24 fev.

44. Silva MJS, Lima FLTL, O’Dwyer G, Osorio-de-Castro CGS. Política de Atenção ao Câncer no Brasil após a criação do Sistema Único de Saúde. R Bras Canc 2017; 63(3):177-187.

45. Kamisetty A, Magennis P, Mayland C, Jack B, Lowec D, Rogers SN. Where do patients treated for oral cancer die? A 20-year cohort study1992-2011. Br J Oral Maxillofac Surg 2015; 53(10):1015-1020.

Artigo apresentado em 27/02/2018

Aprovado em 26/11/2018

Versão final apresentada em 28/11/2018 
